\title{
The Beta-Ray Disintegration
}

A DISCUSSION on the $\beta$-type of nuclear transformation was held by the Royal Society on March 4. Papers were read by Prof. C. D. Ellis, Dr. J. D. Cockeroft, Dr. R. Peierls and Dr. H. O. W. Richardson; a number of other experimental and theoretical workers took part in the discussion. The main centre of interest seemed to be in the correctness or otherwise of the neutrino theory.

Prof. C. D. Ellis, in his opening address, gave a brief account of the development of our knowledge of the $\beta$-ray transformation in recent years. As is generally known, the fundamental difficulty is that the electrons (or positrons) are emitted with a continuous energy distribution although there must be a constant difference between the proper energies of the initial and final nuclei. This anomaly could be explained either by abandoning the conservation laws, or as suggested by Pauli, assuming that another uncharged particle, the neutrino, is emitted in the transformation and shares the available energy with the electron. This latter alternative is now generally favoured.

The neutrino has been given a spin equal to that of the electron, to enable angular momentum to be conserved in the transmutation ; and a mass, which if not zero, is only of the same orcler as that of the electron. A particle with these properties would be very difficult to observe directly, and in fact the many attempts which have been made to detect the neutrino by any ionization it produces have all failed. The hypothesis can at present only be tested by its success in accounting for the features of the $\beta$-ray decay.

One consequence of the neutrino hypothesis can be soen without any detailed theory. The $\beta$-ray spectrum should have a definite upper limit at which the neutrino is emitted with zero kinetic energy, and this end point should be given by the difference between the masses of the particles before and after the transmutation. If these predictions were not fulfilled, the neutrino theory would have to be abandoned.

The evidence on this point was discussed by Dr. Cockcroft. In about six cases of $\beta$-transformation among the light elements, the end point of the spectrum is known with fair accuracy, and the difference between the initial and final masses can be obtained from other transmutations in which energy is certainly conserved. The results are sufficiently definite to show that the mass difference cannot be equated to the mean energy of the $\beta$-ray spectrum, but its identity with the experimentally determined end point is still rather uncertain. However, even this is very useful evidence. It is known that the total energy emission as measured in a calorimeter corresponds to the mean energy of the spectrum. As the change in mass in the transmutation is certainly greater than this, there is a definite surplus of energy which must either have disapjeared or have been carried out of the calorimeter in the form of neutrinos.

Further data on the identity of the end point of the spectrum and the mass difference of the initial and final nuclei would be very important, as this is one of the few points on which the neutrino hypothesis is capable of being disproved. However, the deter. mination of the end point of the spectrum is a matter of some difficulty, because the intensity approaches zero very slowly with increasing energy. The actual end point must be found by extrapolation. Here a theory of the form of the $\beta$-ray spectrum would be very useful. Unfortunately, the most successful theory, that of Uhlenbeck and Konopinski, disagrees with the observed spectrum just in this region, and extrapolations based on it give values for the end points which appear to be too high. In these circumstances, all that can be said is that the experiments do not disagree with the value for the end point given by the mass change in the disintegration.

The detailed mathematical development of the theory cnables it to be compared with other experimental data. We may expect the theory to predict the form of the $\beta$-ray spectrum and how it depends on such parameters as the total energy released and the change of nuclear spin in the transmutation. Also the theory should give the value of the total probability of disintegration, or the decay constant. The general case of $\beta$-decay is one in which the final nucleus may be formed in one of a number of excited states. The $\beta$-ray spectrum is then built up of a number of partial spectra, each with a different end point and intensity. In examining such a spectrum, the experimenter depends on theoretical guidance on these points.

The theoretical aspects of the problem were dealt with by Dr. Peierls. The theory is based on the analogy between the transition of an atom between two stationary states with the emission of a light quantum, and the transmutation of a neutron into a proton with the emission of an electron and a neutrino. A remarkable consequence of this point of view is that it should be possible for a nucleus to be transformed by capturing one of its $K$ electrons and emitting a neutrino-a new type of transmutation which has not yet been observed.

To proceed further, the theoretical worker has to assume some form of interaction between the neutrino and the heavy particles which give it birth. Here he has little to guide him but the demands of relativistic invariance and his æsthetic sense. The most simple and natural assumption was made by Fermi, but this led to a form of the $\beta$-ray spectrum which was definitely too symmetrical about the mean energy. Konopinski and Uhlenbeck remedied this by using a form of interaction which depended on the velocity of the neutrino as well as on its position. At first they appeared to have been very successful. Experiments with the large sources of light radioactive elements now available in the United States gave very satisfactory agreoment with the new theory. A more detailed study has since shown that though the main part of the spectrum was given correctly, the high- and low-energy regions required modification. The evidence on this point was discussed by Dr. Richardson, who showed that whereas fair agreement with experiment was obtained for the low. energy part of the spectrum when account was taken of the Coulomb forces on the electrons, the Konopinski-Uhlenbeck theory gave too high an intensity for the high-energy electrons, in some cases by a factor of fifty. 
Attention has therefore been turned to other possible modifications of the theory. A very wide choice seems to be possible, so that the theory could be made to fit almost any form of the experimental spectrum in several different ways. This elasticity is perhaps an advantage, in that there is no immediate danger of our being forced to abandon the neutrino theory and therefore the conservation laws, but it destroys much of the heuristic value of the theory. For example, although the $\beta$-transmutations can be classified into two groups, corresponding to the two Sargent curves connecting the decay constant with the energy of the end point, it is not known how this classification is connected with the change of nuclear spin in the transmutation.

In conclusion, the general impression given by this discussion was that although the neutrino theory is probably capable of explaining most of the features of the $\beta$-ray transmutation, it scarcely does so in the natural and unforced way which alone would reconcile the physicist to an unobservable particle. However, it remains a working hypothesis useful in directing attention to the points which still require experi. mental work, and in collating the data already obtained.

A. NunN May.

\section{Lightning}

$\mathrm{A}^{\mathrm{N}}$ $\mathrm{N}$ instructive paper on lightning was read on January 7 by B. L. Goodlet, of the Metropolitan-Vickers Electric Co., Ltd., to the Institution of Electrical Engineers. He discussed lightning first as a physical phenomenon and then gave the elementary theory of direct strokes to transmission circuits. He began by saying that although "Lightning" was the title of the Kelvin Lecture given seven years ago, yet the progress made during the last seven years is at least as great as that made during the previous seventy. He awarded due credit to the early pioneers, but modern research on the subject has been so intensive in high-tension laboratories, in the upper atmosphere and in connexion with the tens of thousands of miles of high-tension overhead wires now in continuous operation, that the modern research workers have large sources of experimental data from which they can test theories widely accepted in recent years.

The cumulo-nimbus cloud, or thundercloud, differs from the ordinary rain cloud in having greater vertical dimensions and in the existence of powerful upward air currents. Its formation is associated with convectional instability of the atmosphere, the separation of the electric charge being effected by the resulting upward currents of air. Lightning strokes usually discharge negative electricity into the ground, and consist of a preliminary 'leader' stroke from cloud to earth followed by a powerful return stroke from earth to cloud. Schonland found by means of a Boys camera that each flash is initiated by a streamer which shoots rapidly downwards from the cloud towards the ground, but becomes extinguished before it has travelled more than a fraction of the total distance. About 50 microseconds later, a second streamer follows closely on the track of the first and elongates the channel a little farther. This goes on until an ionized channel is blazed all the way from cloud to earth. The rate of growth of the channel is of the order of 100 kilometres per second.

The maximum current at the ground end of a lightning flash is deduced from the intensity of the magnetic field produced in the vicinity of transmission towers and lightning conductors struck by lightning. About 24,000 magnetic detectors were distributed over the lines of various German electric power companies in 1934. The results showed that the most frequent value of the maximum stroke current lay between 30,000 and 40,000 amperes. Currents greater than 100,000 amperes occur very rarely. In 97 per cent of the cases recorded, the cloud discharged negative electricity to the earth. Presumably therefore the base of the cloud must have been negative. Similar results have been obtained in the United States.

The question has often been discussed as to whether certain localities receive an abnormal pro. portion of lightning strokes. The evidence in favour of localization of lightning flashes comes mainly from the records of electricity undertakings. It has been found, for example, that a certain $80 \mathrm{~km}$. line received 43 strokes, of which 26 fell on one section $6.3 \mathrm{~km}$. in length. Investigation showed that this section of the line was traversed by underground springs. A study of available data has convinced the author that the existence of 'danger spots' struck more frequently for a given storm exposure is a fact.

Some interesting lightning problems occur in connexion with aircraft. Ten cases are known of British aeroplanes having been struck by lightning. In nine out of the ten cases, the wireless aerial was hanging down and was damaged. Moored kite balloons are particularly troubled by phenomena of this nature. When an aeroplane or an airship is flown through a thundercloud, the metal parts of the machine, unless bonded together, acquire very different potentials. Shocks are experienced by persons inside the aircraft when they touch and when they leave go of metal objects.

The earth currents caused by lightning can be a source of great danger. If a current of 50,000 amperes enters the soil at a point and spreads out uniformly in all directions, the voltage drop on the surface as we go away from the point may be very large even at some distance away from the point. The voltage between two points on the earth separated by the length of an animal's stride may be quite sufficient to pass a lethal current up one leg and down another.

The second part of the paper was more technical, being concerned mainly with direct strokes to transmission circuits. In this case rather more than half the direct strokes to a line fall on the conductors in the span between the towers. The final potential of an insulated conductor so struck is probably never less than 2 million volts, and may be sixteen times as great. The insulator impulse spark-over voltage of the most heavily insulated overhead line yet 